Irish Math. Soc. Bulletin

Number 76, Winter 2015, 55-60

ISSN 0791-5578

\title{
A New Characterization of Boolean Rings with Identity
}

\author{
PETER DANCHEV
}

\begin{abstract}
We define the class of nil-regular rings and show that it coincides precisely with the class of boolean rings. We thus give a complete description of these rings.
\end{abstract}

\section{INTRODUCTION}

Throughout this note, let all rings considered be associative, not necessarily commutative a priory, containing an identity element 1 . Since the present paper deals with boolean rings and their generalizations, the condition of having 1 is essential, because finite boolean rings always possess an identity, but this is not in all cases true for infinite boolean rings - e.g., just consider all finite subsets of a given infinite set under the operations of symmetric set difference and intersection.

Furthermore, almost all our notions and notations are standard and follow those from [5]. The other non-classical concepts and terminology will be explained below in detail. For example, a unit $u$ is called unipotent if it is of the form $1+n$, where $n$ is a nilpotent. Also, a ring $R$ is called abelian if all its idempotents are central, that is, they commute with all elements of the ring.

For completeness, we first recall some classical definitions of ring theory. So, a ring $R$ is said to be regular if, for each $a \in R$, there is $x \in R$ such that $a=a x a$, and strongly regular if, for every $a \in$ $R$, there is $x \in R$ such that $a=a^{2} x$. It is well known that the strongly regular rings are exactly the regular rings without nilpotent elements, and also that they are exactly the abelian regular rings (see, e.g., [4] and [7]). It is also a well-known fact that a ring $R$ is strongly regular if and only if each element of $R$ is a product of

2010 Mathematics Subject Classification. 16E50, 16S34, $16 \mathrm{U} 10$.

Key words and phrases. Boolean Rings, Nil-regular rings, Nilpotents, Idempotents.

Received on 5-6-2015; revised 8-6-2015 and 6-11-2015. 
a unit and a central idempotent. Likewise, a ring $R$ is said to be unit-regular if, for each $a \in R$, there exists a unit $u \in R$ such that $a=$ aua. Moreover, it is known that a ring is unit-regular if and only if each of its elements is the product of a unit and an idempotent. Thus strongly regular rings are unit-regular; actually, the element $x$ in the presentation $a=a^{2} x$ can be chosen to be a unit with $a x=x a$ (see, e.g., [3], Theorem 3]).

So, we are ready to state our main tool.

Definition. We shall say that an arbitrary ring $R$ is nil-regular if, for every $r \in R$, there exists a nilpotent $n$ with the property that $r=r(1+n) r=r^{2}+r n r$. Such an element $r$ is also said to be nil-regular.

If the element $r$ can be written as $r=r^{2}(1+n)=r^{2}+r^{2} n$, it is called strongly nil-regular and if this holds for each such $r$, the ring $R$ is called strongly nil-regular as well.

The objective of the present paper is to characterize completely (strongly) nil-regular rings. Surprisingly, we shall show below that these rings do not possess non-trivial nilpotents; thus we ambiguously obtain that they coincide with the classical boolean rings in which each element is an idempotent. In fact, it is obviously seen that every boolean ring is (strongly) nil-regular by taking $n=0$, but the eventual validity of the converse containment is definitely non-trivial. Before showing that it really holds, we will establish an equivalent property of nil-regular rings.

\section{THE MAIN RESUlT}

Proposition 2.1. A ring $R$ is nil-regular if and only if, for any $a \in R$, there exist an idempotent $e$ and a nilpotent $n$ such that $a=e(1+n)$.

Proof. Necessity. Since by definition $a=a(1+n) a$, then we define $e=a(1+n)$ and therefore $a=e a=e a(1+n)(1+n)^{-1}=$ $e(1+n)^{-1}$, where $(1+n)^{-1}$ is of the form $1+t$ for some nilpotent $t$. Moreover, $e^{2}=e \cdot e=a(1+n) a(1+n)=a(1+n)=e$, as required.

Sufficiency. If $a=e(1+n)$, then $a(1+n)^{-1}=e$, so $a(1+$ $n)^{-1} a(1+n)^{-1}=a(1+n)^{-1}$, and hence $a(1+n)^{-1} a=a$, where $(1+n)^{-1}$ is equal to $1+t$ for some nilpotent $t$. 
It is worthwhile noticing that since our basic definition is leftright symmetric, one plainly checks that the last statement may be written as $a=(1+m) f$ for any element $a \in R$ and for some existing nilpotent $m$ and an idempotent $f$.

The next two technical lemmas are referred to in [1] and [2].

Lemma 2.2. If $R$ is a ring with unipotent units, then so is the corner ring eRe for any idempotent $e$.

Proof. Letting $u \in e R e$ be a unit with inverse $v \in e R e$, it is routinely checked that $u+1-e$ is a unit in $R$ with inverse $v+1-e$. Consequently, $u+1-e=1+t$ for some nilpotent $t$, so that $t=$ $u-e \in e R e$ is a nilpotent. We therefore have that $u=e+t$, which is obviously a unipotent, as desired.

Lemma 2.3. For any $n \geq 2$ and any non-zero ring $R$, the matrix ring $\mathbb{M}_{n}(R)$ cannot have unipotent units only.

Proof. Since $\mathbb{M}_{2}(R)$ is isomorphic to a corner ring of $\mathbb{M}_{n}(R)$, in accordance with Lemma 2.2 , it is enough to show that $\mathbb{M}_{2}(R)$ has units other than unipotent ones. To this aim, consider the matrix unit $M=\left(\begin{array}{ll}0 & 1 \\ 1 & 1\end{array}\right)$, with inverse $\left(\begin{array}{cc}-1 & 1 \\ 1 & 0\end{array}\right)$. Since $\left(\begin{array}{ll}1 & 0 \\ 0 & 1\end{array}\right)-\left(\begin{array}{ll}0 & 1 \\ 1 & 1\end{array}\right)=$ $\left(\begin{array}{cc}1 & -1 \\ -1 & 0\end{array}\right)$ is clearly not a nilpotent, because it is again a unit with inverse $\left(\begin{array}{cc}0 & -1 \\ -1 & -1\end{array}\right)$, we deduce that $M$ is not a unipotent, as desired.

We now have all the ingredients needed to prove the promised above new characterization of boolean rings.

Theorem 2.4. Any nil-regular ring is boolean, and conversely. Even more, the following three conditions are equivalent:

(a) $R$ is nil-regular;

(b) $R$ is unit-regular with unipotent units;

(c) $R$ is boolean.

Proof. It is clear that the implications (c) implies (b) implies (a) hold. We will now show that (a) yields (c). To that goal, let (a) be satisfied and we will prove three things about the nil-regular ring $R$ :

(1) Each unit of $R$ is unipotent. 
In fact, given an arbitrary unit $u$, we have $u=u(1+n) u=u$ for some nilpotent $n$, whence $u(1+n)=(1+n) u=1$, implying that $u$ is equal to $(1+n)^{-1}=1+t$, where $t$ is some nilpotent.

(2) $R$ has characteristic 2 .

In fact, as $R$ is regular, its Jacobson radical $J(R)$ must be zero. But since 2 lies in $J(R)$, because $-1=1+n$ for some nilpotent $n$ and hence $2=n$, we deduce that $2=0$, as claimed.

(3) $R$ has no non-zero nilpotents.

In fact, it is easily checked that nil-regular rings $R$ being regular are semi-potent rings (i.e., each left ideal not contained in $J(R)$ contains a nonzero idempotent) with $J(R)=0$, and knowing this let $n>1$. Furthermore, a well-known result of [6] assures us that if $n \in \mathbb{N}$ is such that for any nonzero $a \in R$ with $a^{n}=0$, we have $a^{n-1} \neq 0$, then $R a R$ contains a system of $n^{2}$-matrix units; so $R a R$ will contain a corner ring isomorphic to an $n$ by $n$ matrix ring. But, in view of Lemma 2.2, the property (1) goes down to corner rings, while, if $T \neq 0$ is any ring, then appealing to Lemma 2.3, the matrix $\mathbb{M}_{n}(T)$ cannot have the property of having only unipotent units, so that by (1) it is never nil-regular. This contradiction establishes our assertion.

Using the argumentation above about strongly regular rings, and especially that they do not possess nontrivial nilpotent elements, one may deduce the following:

Proposition 2.5. The following three conditions are equivalent:

(i) $R$ is strongly nil-regular;

(ii) $R$ is strongly regular with trivial units;

(iii) $R$ is boolean.

In particular, nil-regular rings are strongly nil-regular, and vice versa.

In conjunction with Theorem 2.4, concerning the element-wise description, we will illustrate now that there is a nil-regular element which is not an idempotent. In fact, in the matrix ring $\mathbb{M}_{2}(\mathbb{Z})$ one we have $E_{12}=E_{12}\left(1+E_{21}\right) E_{12}$, where one obviously verifies that $E_{21}$ is a nilpotent of index 2 and $E_{12}$ is also a nilpotent of index 2 but not an idempotent, as expected. So, a question which immediately arises is whether or not any strongly nil-regular element is an idempotent. We conjecture that the answer is no, too. 
The ideas presented above can be extended in the following way: In conjunction with [2 one may ask whether or not the rings $R$ for which, for each $r \in R$, there exist an idempotent $e$ and a nilpotent $n$ such that $r(e+n) r=r$ are precisely the nil-clean rings.

We also call a ring $R \pi$-nil-regular if, for each $r \in R$, there exist an integer $i$ and a nilpotent $n$ such that $r^{i}=r^{i}(1+n) r^{i}=r^{2 i}+r^{i} n r^{i}$; such an element $r$ is also called $\pi$-nil-regular. Clearly, all $\pi$-nilregular rings form a subclass of the class of $\pi$-regular rings. Also, one sees that a power of any unit in such a ring is a unipotent.

In this way, if for each $r \in R$ there are an integer $i$ and a nilpotent $n$ such that $r^{i}=r^{2 i}(1+n)=r^{2 i}+r^{2 i} n$, the ring $R$ is called strongly $\pi$-nil-regular; such an element $r$ is also called strongly $\pi$-nil-regular. Evidently, these rings are strongly $\pi$-regular and thus unit-regular.

Recall also that a ring $R$ is said to be $\pi$-boolean if, for every $r \in R$, there exits an integer $i$ such that $r^{2 i}=r^{i}$; such an element $r$ is also called $\pi$-boolean. Apparently, $\pi$-boolean rings are (strongly) $\pi$-nilregular.

So, in accordance with Theorem 2.4, we close with the following question:

Problem 1. Does it follow that (strongly) $\pi$-nil-regular rings do not contain non-trivial nilpotent elements, that is, are (strongly) $\pi$-nil-regular rings exactly the $\pi$-boolean rings?

If the existing nilpotent element in the definition of (strongly) $\pi$ nil-regular rings is unique, we then call such rings uniquely (strongly) $\pi$-nil-regular. So, the next query arises quite naturally:

Problem 2. Characterize uniquely (strongly) $\pi$-nil-regular rings. Are they just the abelian (strongly) $\pi$-nil-regular ones?

Problem 3. Describe the element-wise relationships between $\pi$-nilregular, strongly $\pi$-nil-regular and $\pi$-boolean elements. Is it true that for any element $r$ of a ring $R$ it is strongly $\pi$-nil-regular if and only if there exists an integer $i>0$ such that $r^{i}$ is strongly nilregular? Likewise, is the record of a strongly nil-regular element left-right symmetric?

Acknowledgment: The author of this article is grateful to Professor Yiqiang Zhou for valuable communication in the subject. The 
author is also indebted to the referee for the insightful suggestions made.

\section{REFERENCES}

[1] G. Calugareanu, UU rings, Carpathian J. Math. 31 (2015), 157-163.

[2] P.V. Danchev and T.Y. Lam, Rings with unipotent units, Publ. Math. Debrecen 88 (2016).

[3] G. Ehrlich, Unit-regular rings, Portugal. Math. 27 (1968), 209-212.

[4] K.R. Goodearl, Von Neumman Regular Rings, second edition, Krieger Pub. Co., 1991, 412 pages.

[5] T.Y. Lam, A First Course in Noncommutative Rings, Graduate Texts in Math., Springer-Verlar, 2001.

[6] J. Levitzki, On the structure of algebraic algebras and related rings, Trans. Amer. Math. Soc. 74 (1953), 384-409.

[7] A. Tuganbaev, Rings Close to Regular, Math. Appl., Springer-Verlag, 2002.

Peter Danchev is Professor of Mathematics at Plovdiv and his interests are Abelian Groups and Group Algebras, Associative Rings (both commutative and non-commutative), and Matrix Theory.

(Danchev) Department of Mathematics, Plovdiv State University, Plovdiv 4000, Bulgaria

E-mail address: pvdanchev@yahoo.com 